\title{
The Role of Awake Extracorporeal Membrane Oxygenation as Bridging Therapy for Lung Transplantation: A Retrospective Cohort Study
}

\section{NAM EUN KIM}

Yonsei University College of Medicine https://orcid.org/0000-0003-1817-4415

\section{Song Yee Kim}

Yonsei University College of Medicine

Ah Young Leem

Yonsei University College of Medicine

\section{Youngmok Park}

Yonsei University College of Medicine

\section{Se Hyun Kwak}

Yonsei University College of Medicine

\section{Seung Hyun Yong}

Yonsei University College of Medicine

\section{Kyungsoo Chung}

Yonsei University College of Medicine

\section{Moo Suk Park}

Yonsei University College of Medicine

\section{Young Sam Kim}

Yonsei University College of Medicine

Jin Gu Lee

Yonsei University College of Medicine

Hyo Chae Paik

Yonsei University College of Medicine

Su Hwan Lee ( $D$ HIHOGOGO@yuhs.ac)

Yonsei University College of Medicine https://orcid.org/0000-0002-3487-2574

\section{Research}

Keywords: awake ECMO, extracorporeal membrane oxygenation, intensive care, lung transplantation

Posted Date: August 5th, 2020

DOI: https://doi.org/10.21203/rs.3.rs-52223/v1 
License: (c) (i) This work is licensed under a Creative Commons Attribution 4.0 International License. Read Full License 


\section{Abstract}

Background As lung transplantation (LTx) becomes a standard treatment for end-stage lung disease, bridging to LTx with extracorporeal membrane oxygenation (ECMO) is increasing during waiting time, for either rescue treatment or improving ability to rehabilitation before transplant. This study investigated post-operative outcomes in patients bridging to lung transplantation with ECMO, especially those receiving awake ECMO. Methods In this single-center study, we retrospectively reviewed 241 consecutive LTx patients between October 2012 and March 2019. Among them, 65 patients received ECMO support while waiting for LTx; these patients were analyzed according to their awakeness. Multivaribale logistic regression and Cox proportional hazard models were used to analyze variables associated awake strategy and mortality. Results Thirty-three patients (50.7\%) were awake during bridging ECMO, and 32 patients (49.2\%) were in sedative status. The median age of awake ECMO patients was 59.0 (IQR 54.063.0) years, and $63.1 \%$ of population was male. There were no significant differences between awake and non-awake ECMO patients with respect to age, comorbidities, APACHE II score, ECMO duration and ECMO blood flow. Awake group have better post-operative outcome in terms of statically shorter postoperative intensive care unit (ICU) length of stay (LOS) (awake vs. non-awake, 6 [4-9.5] vs. 16 [6-22], p = 0.004) and longer ventilator free days (VFDs) (awake vs. non-awake, 24 [11.0-25.0] vs. 0 [0.0-14.5], $p=$ 0.001). Furthermore, the awake ECMO group had a significantly lower six-month mortality rate compared to the non-awake group ( $18.2 \%$ vs. $40.6 \%, p=0.045)$. It was independent predictive factor for ability to gait after LTx ([OR] 4.128, 95\% Cl 1.094-15.572, p = 0.036). Conclusions Awake ECMO therapy could be useful for high-risk patients waiting for LTX, and might help shorten ICU LOS and improve survival benefit after LTx. Furthermore, awake ECMO was independent predictive factor for postoperative gaiting.

\section{Background}

Lung transplantation (LTX) is a final treatment option for patients with end-stage lung disease. Since the first successful LTx by Cooper and colleagues in 1983, the number of lung transplants have increased to up to 69,000 cases over the past 30 years. Most LTx are performed in patients with slowly progressive end-stage lung disease; however, some patients need bridging strategies due to severe acute or chronic respiratory failure [1-3].

These issues have increased the interest in bridging strategies for LTx candidates who become too sick to survive until an organ is available. Bridging with veno-venous extracorporeal membrane oxygenation (ECMO) had reduced the requirement for refractory hypercapnia or hypoxia with a mechanical ventilator [4]. It was considered a relative contraindication to lung transplantation in the 1970s and 1980s, due to poor perioperative outcomes and many complications [5, 6]. However, as ECMO-related techniques and experience improved, literature on pre-transplant ECMO showed postoperative benefits in high-risk candidates; accordingly, the proportion of patients receiving bridging ECMO is increasing [7-11].

Furthermore, a concept called "awake ECMO" has been used for bridging. ECMO could replace the role of a mechanical ventilator, as candidates can spontaneously breathe even after extubation with early 
tracheostomy. Such a strategy not only enables the patient avoid complications related to mechanical ventilation, sedation, and immobilization, but also enables active treatment participation and pulmonary rehabilitation, as well as the optimization of nutritional support. Consequently, it extends the candidacy of recipients and helps them maintain their physical condition whilst waiting for $\operatorname{LTx}[4,12,13]$.

However, the use of awake ECMO is associated with problems. Cannula displacement, patient discomfort, pain, and anxiety could occur in conscious patients in the intensive care unit (ICU) [14]. In addition, there are no uniform principles about patient selection, cannulation approach, inclusion and exclusion criteria for the bridging of ECMO at high-volume centers [2].

In this study, we evaluate postoperative outcomes and survival of LTx candidates with pre-transplant ECMO bridging, with a focus on the role of awake ECMO. The primary outcomes were three- and sixmonth mortality, and the secondary outcomes were post-LTx outcomes such as postoperative gait, ventilator free days (VFDs), ICU length of stay, and hospital length of stay (LOS). Also, further analysis was done according to the quality of liberation from mechanical ventilation in patients undergoing awake ECMO.

\section{Methods}

\section{Study population}

This was a retrospective, single-center, seven-year cohort study in consecutive patients receiving LTx in South Korea, between October 2012 and June 2019. During the study period, 241 patients received LTX. Fourteen patients were excluded due to the following exclusion criteria: five patients were under the age of 18 years, five patients underwent co-transplantation of the lung and another organ, two underwent LTx with off-pump coronary artery bypass, and two patients had re-transplantation (Fig.1 Study design). The study protocol was approved by the institutional review board of Severance Hospital (approval no. 42019-1294). It adhered to the principles of the Declaration of Helsinki (2000) and the Declaration of Istanbul (2008).

All patients used ECMO during transplantation surgery and were administered induction immunosuppression therapy with high dose corticosteroids: methylprednisolone $500 \mathrm{mg}$ during the operation, and $0.5 \mathrm{mg} / \mathrm{kg} /$ day for three days after the operation. Triple immunosuppression therapy, such as prednisolone, tacrolimus, and mycophenolate mofetil, was used for maintaining immunosuppression after transplantation. Patients who received bridged ECMO in 2019 received basiliximab $20 \mathrm{mg}$ at the time of LTx and started taking tacrolimus after seven days. Ganciclovir and itraconazole were used all recipients until six months after their operation. Lifelong trimethoprim/sulfamethoxazole was used in all recipients to prevent Pneumocystis jirovecii after LTX.

\section{Data collection and definition}


All data of enrolled patients were collected from the electronic medical records of the hospital. Patient data, including demographic data, laboratory values, comorbidities, and transthoracic echocardiogram parameters before LTx and after LTx were collected. ECMO flow was detected at $24 \mathrm{~h}$ after cannulation.

To describe the patient's status when using mechanical ventilation or ECMO therapy, the acute physiology and chronic health evaluation II (APACHE II) score was collected at the time of admission to medical ICU for bridging therapy.

VFDs are usually calculated using one point for each day during the measurement period that a patient is both alive and free of mechanical ventilation [15]. In our study, patients in the post-LTx period who could breathe for at least $24 \mathrm{~h}$ without assistance of a mechanical ventilator or home ventilator were regarded as having a ventilator-free status. For example, if a patient was extubated on postoperative day two and remained alive and free of ventilatory support for the subsequent 28 days, their VFDs score would be 26 .

The quality of rehabilitation during the pre-LTx and post-LTx periods were checked. We examined the patients' range of motion and functional mobilization levels, including assessment of side rolling at the bedside, come to sit, sit to stand, standing unsupported, and walking in the pre-LTx period. After LTx period, we examined the walking ability and time spent walking.

The six-minute walking test (6MWT) was performed in patients who could ambulate at one, three, and six months after LTx in our center. The 6MWT was conducted in line with the American Thoracic Society guidelines [16]. The assessor recorded baseline oxygen saturation levels, heart rate, and the Borg scale rating for dyspnea and fatigue at the beginning of walking and at the end of the test, which was performed in a $30 \mathrm{~m}$ hallway. The distance covered in six minutes was recorded.

\section{Awake ECMO and physical rehabilitation}

Potential candidates for ECMO support include patients already listed for lung transplantation who have developed respiratory failure even with small movements, such as eating, drinking, and coughing, despite high-flow oxygen support via nasal cannula. Also, patients with acute respiratory failure and poor gas exchange with use of a mechanical ventilator were eligible for salvage treatment, as were patients who had been transferred to our tertiary center due to the impossibility of ECMO weaning locally, waiting for LTX.

Since bi-caval dual lumen ECMO catheters have not been used in South Korea, most patients who need ECMO undergo a right jugular-femoral venous configuration. Some patients who developed right heart failure due to lung problems were changed to veno-arterial-venous cannulation.

After cannulation, all patients received systemic anticoagulation through the intravenous administration of heparin, in order to maintain an activated clotting time of 160-180 seconds. Blood flow was managed to a target partial pressure of oxygen $>60 \mathrm{mmHg}$, while sweep gases were managed to maintain $\mathrm{pH}$ over 7.35 and a partial pressure of carbon dioxide $\leq 45 \mathrm{mmHg}$. The mechanical ventilation settings followed an ultra-protective strategy (predicted body weight, $\leq 4 \mathrm{ml} / \mathrm{kg}$ ) and infusions of sedative and analgesic 
drugs were tapered. If the patient was agitated, low dose dexmedetomidine was used to maintain a Richmond Agitation and Sedation Scale (RASS) score of 0-1 point.

Patients underwent attempted extubation within 24 to $48 \mathrm{~h}$ of the initiation of ECMO if they had stable gas exchange and hemodynamic status. If the weaning process was predicted to be difficult, early tracheostomy was performed within 48 to $72 \mathrm{~h}$ for a spontaneous breathing trial.

The majority of the literature defines awake ECMO as liberation from invasive mechanical ventilation by extubation or early tracheostomy with spontaneous breathing $[4,13,17]$. However, some recipients who cannot wean totally from the ventilator need mechanical ventilator support modes such as continuous positive airway pressure (CPAP); these are different from completely awake (extubated) patients. However, these differences were not reflected in previous other studies.

In our study, we define awake ECMO as patients who could maintain their awareness with a RASS score of 0-1 point, free from a mechanical ventilator, and spontaneously breathing while extubated or with early tracheostomy and partial ventilator support. We further subclassified the awake ECMO group into a 'totally awake group' and a 'partially awake group', due to different efforts in the weaning process. In the totally awake group, the previous procedure was easily performed, so patients could breathe independently within $48 \mathrm{~h}$. In the partially awake ECMO group, the weaning process was difficult but did not fail completely, with the patients consequently requiring ventilatory support such as CPAP for spontaneous breathing.

Awake ECMO treatment was considered a failure if patients met the following criteria: severe dyspnea and respiratory rate more than 30 breaths/min due to hypoxemia associated with uncontrolled ECMO alone, agitation for dyspnea, or an inability to clear airway secretions.

Early physical therapies and mobilization were achieved in patients receiving awake ECMO. Physical therapies ranged from passive range of motion of the limb at the bedside to sitting at the edge of the bed and standing at the bedside. If previous mobilization was tolerable with ECMO in the absence of desaturations, tachypnea, marching with the walker, or walking with assistance could be attempted if possible. However, in our center, there were no ambulatory patients during the maintenance of ECMO. The maximum rehabilitation activities observed were standing and bedside cycle ergometer. During awake ECMO, patients were receiving nasal cannula or high-flow nasal cannula oxygen therapy.

\section{Statistical Analysis}

The statistical analyses were performed using the SPSS software, version 25.0 (IBM Corp., Armonk, NY, USA). Continuous variables are presented as median (interquartile range [IQR]) and categorical variables are presented as number and percentages. An assessment for normal distribution of the data was performed.

Medians were analyzed using the Mann-Whitney $\mathrm{U}$ test and the nonparametric Wilcoxon-W test. All reported P-values are two-sided. Categorical variables were analyzed using the Chi-squared test or 
Fisher's exact test. Kaplan-Meier statistics were used to estimate survival rates with statistical significance assessed by log rank analysis.

Factors associated with survival were determined using Cox regression analysis. To confirm the assumption of proportionality, time-dependent covariate analysis was used. The time-dependent covariate was not statistically significant, suggesting that the proportional hazards assumption was reasonable. Estimates for hazard ratios (HR) and 95\% confidence intervals ( $\mathrm{Cl}$ ) were obtained. For all analyses, P-values less than 0.05 were considered statistically significant.

\section{Results}

\section{Baseline characteristics of the patients receiving bridged ECMO according to the awake strategy}

In this study, among 227 patients awaiting LTx, 65 (28.6\%) were candidates who needed bridging with ECMO. The mortality associated with bridged ECMO was $46.3 \%$, which was not inferior to that of nonbridged ECMO (40.2\%), despite the deteriorating condition in these patients and the unfeasibility of gas exchange with mechanical ventilation. $(p=0.244)$ (Supplement Fig. 1, Kaplan-meier survival curves of LTx patients stratification by bridging ECMO).

A total of 65 patients were treated with ECMO during the bridging period; their median age was 57.0 (IQR 49.5-63.0) years, and 63.1\% of the population was male. The main indication for LTx was idiopathic pulmonary fibrosis $(n=31)$, followed by connective tissue disease-associated interstitial lung disease $(n=11)$. The proportion of awake ECMO patients among the overall LTx population has gradually increased over the past few years in our center (Fig. 2 Percentage of Awake ECMO and ECMO bridging in severance hospital).

Thirty-three patients (50.7\%) underwent awake ECMO support, and 32 patients (49.2\%) underwent ECMO support with sedation and mechanical ventilation (non-awake). Table 1 shows the lack of a significant difference in age, comorbidities, and APACHE II score between the two groups. In the awake group, median ECMO blood flow was 3,100 ml, and the median duration of ECMO while waiting for LTx was 13 days; these parameters were not significantly different in the non-awake group.

Table 1. Baseline characteristics between non-awake ECMO and awake ECMO patients 


\begin{tabular}{|c|c|c|c|}
\hline & $\begin{array}{c}\text { Non-Awake ECMO } \\
(\mathrm{n}=32)\end{array}$ & $\begin{array}{c}\text { Awake ECMO } \\
(\mathrm{n}=33)\end{array}$ & $p$ \\
\hline Age, years, median (IQR) & $54.5(49.0-61.3)$ & $59(54.0-63.0)$ & 0.086 \\
\hline Male, gender, n (\%) & $15(46.9)$ & $26(78.8)$ & 0.008 \\
\hline BMI, $\mathrm{kg} / \mathrm{m}^{2}$, median & $22.1(18.6-25.2)$ & $20.1(17.6-22.3)$ & 0.288 \\
\hline Primary lung disease, $\mathrm{n}(\%)$ & & & 0.135 \\
\hline IPF & $13(40.6)$ & $18(54.5)$ & \\
\hline CTD-related ILD & $4(12.5)$ & $7(21.2)$ & \\
\hline AIP & 5 (15.6) & $0(0)$ & \\
\hline BO after PBSCT & $1(3.1)$ & $2(6.1)$ & \\
\hline Other & $9(28.1)$ & $6(18.2)$ & \\
\hline \multicolumn{4}{|l|}{ Comorbidities, n (\%) } \\
\hline Hypertension & $10(31.3)$ & $6(18.2)$ & 0.221 \\
\hline Diabetes mellitus & $9(28.1)$ & $10(30.3)$ & 0.847 \\
\hline Pulmonary hypertension & $18(56.3)$ & $17(51.5)$ & 0.702 \\
\hline \multicolumn{4}{|l|}{ TTE parameters before LTx } \\
\hline EF, median, \% (IQR) & $61(56.0-66.0)$ & $63(56.0-69.5)$ & 0.416 \\
\hline E/E', median (IQR) & $11(8.0-12.0)$ & $10(8.0-12.0)$ & 0.683 \\
\hline RVSP, median, mmHg (IQR) & $48(30.75-61.0)$ & $50(37.5-57.5)$ & 0.783 \\
\hline TAPSE, median, cm (IQR) & $2(1.4-2.2)$ & $1.75(1.29-1.85)$ & 0.5 \\
\hline TDI, median, (IQR) & $11(9.0-13.0)$ & $11.2(8.25-13.25)$ & 0.765 \\
\hline APACHE II score, median (IQR) & $21(17.0-28.0)$ & $19(13.0-25)$ & 0.133 \\
\hline \multicolumn{4}{|l|}{ Bridging parameters } \\
\hline ECMO Blood flow, mL (IQR) & $3175(2815.0-3565.0)$ & $3100(2790.0-3570.0)$ & 0.637 \\
\hline ECMO duration, days (IQR) & $10.0(4.5-19.0)$ & $13.0(8.0-19.0)$ & 0.290 \\
\hline \multicolumn{4}{|l|}{ Post-transplant TTE parameters } \\
\hline EF, median, \% (IQR) & $63(58.5-68.0)$ & $64(61.0-68.0)$ & 0.374 \\
\hline E/E', median (IQR) & $11(8.0-11.0)$ & $10(7.0-12.0)$ & 0.92 \\
\hline RVSP, median, mmHg (IQR) & $33(27.0-39.5)$ & $33(27.0-39.5)$ & 0.426 \\
\hline TAPSE, median, cm (IQR) & $1.58(1.32-1.89)$ & $1.58(1.32-1.89)$ & 0.613 \\
\hline TDI, median (IQR) & $11(10.0-13.0)$ & $11(10.0-13.0)$ & 0.138 \\
\hline Ability to gait after LTx & $18(56.3)$ & $28(84.8)$ & 0.011 \\
\hline
\end{tabular}




\begin{tabular}{lccc} 
Time to gaiting, days (IQR) & $26.5(14.0-31.0)$ & $18(10.0-30.5)$ & 0.25 \\
\hline 6MWT, m (IQR) & $180(127.5-295.0)$ & $280(185-350)$ & 0.112 \\
\hline VFDs, days (IQR) & $0(0.0-14.5)$ & $24(11.0-25.0)$ & 0.001 \\
\hline ICU LOS, days (IQR) & $16(6-22)$ & $6(4-9.5)$ & 0.001 \\
\hline Hospital LOS, days (IQR) & $73(58.5-87.5)$ & $70.5(56.0-112.0)$ & 0.606 \\
\hline 3M mortality, n (\%) & $7(22)$ & $3(9)$ & 0.152 \\
\hline $6 \mathrm{M}$ mortality, n $(\%)$ & $13(40.6)$ & $6(18.2)$ & 0.045
\end{tabular}

Data are presented as numbers (percentages) or median

Abbreviations: 6MWT, 6-minute walk test; AIP, acute interstitial pneumonia; BMI, body mass index; BO after PBSCT, bronchiolitis obliterans after peripheral blood stem cell transplantation; CTD, connective tissue disease; ECMO, extracorporeal membrane oxygenation; EF, ejection fraction; ICU, intensive care unit; ILD, interstitial lung disease; IMV, invasive mechanical ventilation; IPF, idiopathic pulmonary fibrosis; LOS, length of stay; LTx, lung transplantation; RVSP, right ventricular systolic pressure; TAPSE, tricuspid annular plane systolic excursion; TDI, tissue Doppler imaging; TTE, transthoracic echocardiography; VFDs, ventilator free days.

The proportion of males in the awake group was higher than in the non-awake group $(78.8 \%$ vs. $46.9 \% p$ $=0.008$ ). Awake ECMO patients had a significantly shorter median ICU stay and longer duration of VFDs compared to the non-awake ECMO group (ICU length of stay, 6 [4-9.5] vs. 16 [6-22], $p=0.001$; VFDs, 24 [11.0-25.0] vs. 0 [0.0-14.5], $p=0.001)$. Furthermore, more patients were able to gait after LTx in the awake group compared to the non-awake group $(84.8 \% v s .56 .3 \%, p=0.011)$.

\section{Mortality}

The six-month mortality rate of bridged ECMO patients according to awake and non-awake status using the Kaplan-Meier analysis was 18.2\% (6/33) and 40.6\% (13/32), respectively (log-rank test, $p=0.044$; Fig. 3 Kaplan-meier survival curves of bridged ECMO patients stratification by Awake strategy). Furthermore, we analyzed risk factors influencing the six-month mortality using a Cox model including the variables which were meaningful in the univariate analysis. Postoperative gait was an independent predictive factor for six-month mortality adjusted for age, gender, and body mass index (Postoperative gait; HR, 0.060, 95\% Cl, 0.023-0.162, $p<0.001$; Table 2).

Table 2. Cox regression for awake strategy and six-month mortality adjusting for various prognostic factors 


\begin{tabular}{lcc}
\hline \multirow{2}{*}{ Variables } & \multicolumn{2}{c}{ Multivariable } \\
\cline { 2 - 3 } & $0.979(0.939-1.021)$ & 0.316 \\
\hline Age & $0.697(0.317-1.529)$ & 0.368 \\
Sex, female & $0.990(0.918-1.068)$ & 0.798 \\
\hline BMI & & \\
\hline Postoperative gait & $0.060(0.023-0.162)$ & $<0.001$ \\
\hline Awake ECMO & $0.960(0.407-2.266)$ & 0.926 \\
\hline
\end{tabular}

Abbreviations: BMI, body mass index; CI, confidence interval; ECMO, extracorporeal membrane oxygenation; HR, hazard ratio.

\section{Factors affecting gaiting after LTX}

Based on the previous results, we performed an analysis to identify factors associated with gaiting after LTx in the bridged ECMO group. In multivariate logistic analysis, four factors including age, gender, BMI, and awake status were adjusted for in the analysis of gait after LTx (Table 3). Awake ECMO (odd ratio [OR] 4.128, 95\% Cl 1.094-15.572, $p=0.036$ ) was an independent predictive factor for ability to gait success after $\mathrm{LTX}$, whereas high $\mathrm{BMI}\left(>25 \mathrm{~kg} / \mathrm{m}^{2}\right)(\mathrm{OR} 0.207, \mathrm{Cl} 0.052-0.827, p=0.026)$ were risk factors for gait failure.

Table 3. Logistic regression for post-lung transplantation gait

\begin{tabular}{lcccc}
\hline & \multicolumn{2}{c}{ Univariable } & & Multivariable \\
\cline { 2 - 5 } Variables & OR (95\% CI) & $P$ & OR (95\% CI) & $P$ \\
\cline { 2 - 5 } & $0.993(0.937-1.051)$ & 0.799 & $0.979(0.918-1.044)$ & 0.518 \\
Age & $3.490(1.146-10.635)$ & 0.028 & $2.163(0.611-7.657)$ & 0.232 \\
\hline BMI $\left(\mathrm{kg} / \mathrm{m}^{2}\right)(18.5-24.9)$ & $0.464(0.087-2.492)$ & 0.371 & & \\
\hline BMI $\left(\mathrm{kg} / \mathrm{m}^{2}\right)(>25)$ & $0.095(0.016-0.580)$ & 0.011 & $0.207(0.052-0.827)$ & 0.026 \\
\hline Diabetes mellitus & $1.815(0.513-6.419)$ & 0.355 & & \\
\hline Pulmonary hypertension & $0.793(0.270-2.334)$ & 0.674 & & \\
\hline ECMO duration & $1.021(0.961-1.084)$ & 0.502 & & \\
\hline Awake ECMO & $4.356(1.338-14.180)$ & 0.015 & $4.128(1.094-15.572)$ & 0.036 \\
\hline
\end{tabular}

Abbreviations: BMI, body mass index; CI, confidence interval; ECMO: extracorporeal membrane oxygenation; OR, odds ratio. 


\section{Subgroup analysis of postoperative outcome and survival in awake patients according to the qualification of awake}

We divided the awake group into two further groups according to whether the awake strategy was fully maintained or not. By our definition, 25 patients $(75.7 \%)$ were classified as totally awake, and eight patients $(24.2 \%)$ were partially awake, requiring ventilatory support such as CPAP to maintain spontaneous breathing.

There were no significant differences with respect to age, gender, primary lung disease, or comorbidities between the two groups (Table 4). The median duration of ECMO support in the partially awake group was longer (17.5 days vs. 13.0 days), but this difference was not statistically significant. The median APACHE II score was statistically higher in the partially awake group (26.0 vs. 18.0, $p=0.001)$.

Table 4. Subgroup analysis in the awake ECMO group according to quality of awake 


\begin{tabular}{|c|c|c|c|}
\hline & $\begin{array}{l}\text { Totally awake group } \\
\qquad(\mathrm{n}=25)\end{array}$ & $\begin{array}{l}\text { Partially awake group } \\
\qquad(\mathrm{n}=8)\end{array}$ & $P$ \\
\hline Age, years, median (IQR) & $59.0(55.0-63.0)$ & $59.5(56.0-62.5)$ & 0.801 \\
\hline Male, gender, $\mathrm{n}(\%)$ & $20(80.0)$ & $6(75.0)$ & 1.000 \\
\hline BMI, $\mathrm{kg} / \mathrm{m}^{2}$, median, IQR & $19.3(17.26-23.23)$ & $22.44(20.78-25.75)$ & 0.101 \\
\hline Primary lung disease, $\mathrm{n}(\%)$ & & & 0.378 \\
\hline IPF & $14(56.0)$ & $4(50.0)$ & \\
\hline CTD related ILD & $4(16.0)$ & $3(37.5)$ & \\
\hline AIP & $0(0)$ & $0(0)$ & \\
\hline BO after PBSCT & $2(8.0)$ & $0(0)$ & \\
\hline Other & $5(20)$ & $1(12.5)$ & \\
\hline \multicolumn{4}{|l|}{ Comorbid conditions, n (\%) } \\
\hline Hypertension & $5(20.0)$ & $1(12.5)$ & 1.000 \\
\hline Diabetes mellitus & $6(24.0)$ & $4(50.0)$ & 0.205 \\
\hline Pulmonary hypertension & $12(48.0)$ & $5(62.5)$ & 0.688 \\
\hline \multicolumn{4}{|l|}{ TTE parameters, before LTx } \\
\hline EF, median, \% (IQR) & $62.0(55.0-67.0)$ & $67.0(58.5-74.5)$ & 0.135 \\
\hline E/E', median, \% (IQR) & $10.0(8.27-12.0)$ & $9.0(8.0-10.0)$ & 0.396 \\
\hline RVSP, median, mmHg (IQR) & $49.0(40.0-53.0)$ & $58.0(38.5-67.5)$ & 0.178 \\
\hline TAPSE, median, cm (IQR) & $1.75(1.30-2.2)$ & $1.35(0.8-1.9)$ & 0.324 \\
\hline TDI, median, (IQR) & $11.0(8.1-12.5)$ & $12.0(11.0-13.0)$ & 0.515 \\
\hline APACHE II score, median (IQR) & $18.0(13.0-19.0)$ & $26.0(22.0-28.5)$ & 0.001 \\
\hline \multicolumn{4}{|l|}{ Bridging parameters } \\
\hline ECMO blood flow, mL (IQR) & $3270.0(3100.0-3520.0)$ & $3270.0(3100.0-3520.0)$ & 0.208 \\
\hline ECMO duration, days (IQR) & $13.0(8.0-16.0)$ & $17.5(10.0-26.0)$ & 0.165 \\
\hline \multicolumn{4}{|l|}{ Post-transplant parameters } \\
\hline EF, median, \% (IQR) & $65.0(62.0-69.0)$ & $61.0(59.0-63.0)$ & 0.074 \\
\hline E/e' ratio, median (IQR) & $9.5(7.0-12.5)$ & $11.0(8.0-12.0)$ & 1.000 \\
\hline RVSP, median, mmHg (IQR) & $33.0(29.0-39.0)$ & $33.0(23.0-43.0)$ & 0.972 \\
\hline TAPSE, median, cm (IQR) & $1.50(1.30-1.75)$ & $1.92(1.4-2.11)$ & 0.126 \\
\hline TDI, median (IQR) & $11.0(10.0-13.0)$ & $11.0(10.0-12.5)$ & 0.813 \\
\hline Ability to gait after LTx, n (\%) & $23(92.0)$ & $5(62.5)$ & 0.078 \\
\hline
\end{tabular}




\begin{tabular}{llll} 
Time to gait, days (IQR) & $17.0(10.0-28.5)$ & $28.0(22.0-41.0)$ & 0.055 \\
\hline 6MWT, m (IQR) & $255.0(180.0-340.0)$ & $330.0(255.0-345.0)$ & 0.706 \\
\hline Pre-op mobilization, n (\%) & & & 0.316 \\
\hline Bedside & $14(56.0)$ & $6(75.0)$ & \\
\hline Sitting & $7(28.9)$ & $1(12.5)$ & 0.010 \\
\hline Standing & $4(16.0)$ & $1(12.5)$ & 0.009 \\
\hline VFDs, days (IQR) & $24.0(20.0-26.00)$ & $5.0(0.0-15.0)$ & 0.045 \\
\hline ICU LOS, days (IQR) & $6.0(4.0-8.0)$ & $16.0(10.5-27.5)$ & 0.083 \\
\hline Hospital LOS, days (IQR) & $70.5(56.0-116.5)$ & $113.0(108.50-216.00)$ & 0.094 \\
\hline 3M mortality, n (\%) & $1(0.4)$ & $2(25)$ & 0.021 \\
\hline 6 GM mortality, n (\%) & $3(12.0)$ & $3(37.5)$ & $5(62.5)$ \\
\hline Overall mortality, n (\%) & $5(20.0)$ & & \\
\hline
\end{tabular}

Data are presented as numbers (percentages) or median

Abbreviations: 6MWT, 6 minute walk test; AIP, Acute interstitial pneumonia; BMI, body mass index; BO after PBSCT, Bronchiolitis obliterans after peripheral blood stem cell transplantation; CTD, Connective tissue disease; ECMO, extracorporeal membrane oxygenation; EF, ejection fraction; ICU, Intensive care unit; ILD, Interstitial lung disease; IMV, Invasive mechanical ventilation; IPF, Idiopathic pulmonary fibrosis; LOS, Length of stay; LTx, Lung transplantation; PEEP, Positive end expiratory pressure; RVSP, Right ventricular systolic pressure; TAPSE, Tricuspid annular plane systolic excursion; TDI, Tissue Doppler Imaging; TTE, Trans thoracic echocardiography; VFDs, Ventilator free days.

In the totally awake group, sitting or standing status were more easily achieved than in the partially awake group, but this difference was not statistically significant. The totally awake group had statistically significant improvements in postoperative outcomes and overall survival compared to the partially awake group. ICU LOS after surgery and hospital LOS were significantly shorter in the totally awake group compared to the partially awake group (ICU LOS, 6.0 vs. 16.0, $p=0.025$; hospital LOS 70.5 vs. 113.0, $p=0.025$ ). The duration of VFDs after LTx was significantly longer in the totally awake group (24.0 vs. 5.0, $p=0.010$ ). Furthermore, Kaplan-Meier curves showed a higher overall mortality rate in the partially awake ECMO group compared to the totally awake ECMO group (log-rank test, $p=0.021$; Supplementary Fig. 2 Kaplan-meier survival curves of Awake ECMO patients stratification by totally and partially awake).

\section{Discussion}

Our study showed that the survival of bridged ECMO patients was not inferior to that of non-bridged ECMO patients, and the number of patients receiving bridged ECMO has increased in the past few years. Among the bridged ECMO group, the awake ECMO patients had a significantly higher six-month survival 
compared to the non-awake group using Kaplan-Meier survival analysis, and awake ECMO independently predicted postoperative gait in multivariate analysis.

The main finding of our study is that awake strategy statically improve physical ability to gait after lung transplantation, which affects 6-month survival outcome. Awake approach would minimize ICU-related complications associated with sedation, intubation, prolonged mechanical ventilation and critical illness myopathy. [14, 18-20]

First, the cessation of sedation and being conscious could avoid prolonged immobilization and facilitate pulmonary rehabilitation. Several studies have shown the mechanisms of how immobility can cause muscle weakness. Immobility due to critical illness leads to decreased muscle protein synthesis and increased muscle catabolism, leading to decreased muscle mass [21]. It can lead to a pro-inflammatory state, increasing reactive oxygen species that induce the oxygenation of myofilaments, resulting in contractile dysfunction and muscle atrophy $[22,23]$.

LTx recipients usually have exercise intolerance and long-term inactivity due to dyspnea, consequently leading to peripheral muscle weakness [24]. Also, immunosuppression therapy could delay recovery of quadriceps strength, making it even harder to recover to the patient's pre-operative physical condition [25, 26]. Many patients suffer from substantial muscle dysfunction, reduced physical capacity, and a decline in health-related quality of life $[19,20]$.

Second, maintaining respiratory muscle tone could help ventilation to even dependent parts of the lung, leading to optimal ventilation-perfusion matching after transplantation [14]. Awake patients with spontaneous breathing can move their diaphragm, preventing muscle atrophy [27]. Goligher et al. reported that diaphragm atrophy during mechanical ventilation strongly impacts clinical outcomes, and decreased muscle thickness was associated with lower rates of independence from ventilatory support [28].

Third, being consciousness allows the patient to communicate with nursing staff, medical staff, and their family, and encourages therapy during the waiting period for LTx [14].

However, survival rates in our study were slightly lower compared to previous studies. $[4,13,17]$. In the Columbia group, survival rates up to $92.5 \%$ have been reported [13], and in the Hannover group, survival at six months in the awake ECMO group is $80 \%$ compared to $50 \%$ in the mechanical ventilation group [4]. It could be explained by different policies regarding de-listing criteria after the bridging of ECMO at each center, a variety of cannulation approaches, and different lung allocation systems.

In the Columbia group, slightly strict de-listing criteria during waiting time was observed. In their study, among 72 patients who received awake ECMO, only $55.6 \%$ patients who were able to gait and in a good condition received LTx; among them, $92.5 \%$ survived to discharge [13].

Also, the cannulation technique in our study was different from other studies. In the Cleveland group, advances in cannula design that facilitate gait (double lumen single cannula) have been used, and 
positioning the cannulas in the upper extremities helping gait; in their cohort, 26\% (5 of 19) were successfully gait during awake ECMO [29]. Unfortunately, double lumen single catheters are not available in South Korea, and therefore we had no alternative to femoral catheterization, which may interfere with gait during awake ECMO.

Furthermore, many other countries have organ allocation systems regulated by their government, following the principles of justice and medical utility to achieve the best matching. For example, in the USA the lung allocation score is used to prioritize waiting list candidates based on a combination of waitlist urgency and the probability of post-transplant survival. In Japan, bridged ECMO patients, and those older than 60 years are not added to LTx waiting lists, because poor graft survival is predicted even though their urgency is high. However, in South Korea, organ allocation is governed primarily by the urgency of a patient's status. [30].

Despite these different policies, cannulation approaches, and lung allocation systems, we could find that the strengthen of awake strategy in bridging to LTx patients, reduce complications, maintain or improve physical ability, and that these factors influence LTx outcomes. Furthermore, in totally awake group, survival rate was up to $80 \%$.

There are several limitations to our study. It was a retrospective observational study in a single tertiary center in Asia. However, to the best of our knowledge, this is the first report of an Asian cohort undergoing awake ECMO in LTx. Most previous studies were performed in North America and Europe, and their results are difficult to generalize on account of racial and environmental differences. Therefore, this study will be helpful in understanding LTx in Asians. Second, due to short follow-up duration of approximately six months, there was a lot of censored data. Therefore, well-designed prospective studies are needed to evaluate the long term results and the optimal use of awake ECMO when used as a bridge to LTx. In addition, we focused on the analysis of the role of an awake ECMO strategy, and the results of this study may be helpful to the management of ECMO, when considering the higher mortality rate and the increase of bridged ECMO use among LTx candidates in South Korea [31].

\section{Conclusions}

Awake ECMO could be useful strategy in patients waiting for LTX and may be helpful in shortening ICU stays and improving the survival benefit after LTx. Furthermore, awake ECMO could be helpful in improving postoperative mobilization and physical activity.

\section{List Of Abbreviations}

6MWT, 6-minute walk test; AIP, acute interstitial pneumonia; APACHE II, acute physiology and chronic health evaluation II; BMI, body mass index; BO after PBSCT, bronchiolitis obliterans after peripheral blood stem cell transplantation; $\mathrm{Cl}$, confidence interval; CPAP, continuous positive airway pressure; CTD, connective tissue disease; ECMO, extracorporeal membrane oxygenation; EF, ejection fraction; ICU, 
intensive care unit; ILD, interstitial lung disease; IMV, invasive mechanical ventilation; IPF, idiopathic pulmonary fibrosis; LOS, length of stay; LTx, lung transplantation; PEEP, positive end expiratory pressure; RASS, Richmond Agitation and Sedation Scale; RVSP, right ventricular systolic pressure; TAPSE, tricuspid annular plane systolic excursion; TDI, tissue Doppler imaging; TTE, transthoracic echocardiography; VFDs, ventilator free days.

\section{Declarations}

Ethics approval and consent to participate

The study protocol was approved by the Institutional Review Board (IRB) of Severance Hospital (IRB number: 4-2019-1294). All methods were performed in accordance with the relevant guidelines and regulations. Informed consent was waived by the IRB because of the study's retrospective nature. Consent for publication

Not applicable

Availability of data and materials

The datasets generated during and/or analyzed during the current study are not publicly available due to our IRB policy but are available from the corresponding author upon reasonable request.

Competing interests

The authors declare that they have no competing interests.

Funding

This work was supported by the National Research Foundation of Korea (NRF) grant funded by the Korea government (MSIT) (grant number NRF-2018R1C1B6004282).

Authors' contributions

All authors have made substantial contribution to the paper. Authorship was in accordance with International Committee of Medical Journal Editors (ICMJE,http://www.icmje.org) recommendations: NEK participated in the study conception and design, acquisition of data, analysis and interpretation of data, and drafting the manuscript. SYK, AYL, YMP, SHG, SHY, KSC, MSP, YSK, JGL, HCP helped in the study conception and design, helped in writing article and participated for important intellectual content. SHL participated in study conception and design, analysis and interpretation of data, revising the manuscript critically for important intellectual content, and study supervision. All authors approved final version of the article.

Acknowledgements 
This work was supported by the National Research Foundation of Korea (NRF) grant funded by the Korea government (MSIT) (grant number NRF-2018R1C1B6004282).

\section{References}

1. Shah PD, Orens JB. Guidelines for the selection of lung-transplant candidates. Curr Opin Organ Transplant. 2012;17:467-73.

2. Mattar A, Chatterjee S, Loor G. Bridging to Lung Transplantation. Crit Care Clin. 2019;35:11-25.

3. Cooper JD, Pearson FG, Patterson GA, Todd TRJ, Ginsberg RJ, Goldberg M, DeMajo WAP. Technique of successful lung transplantation in humans. The Journal of Thoracic and Cardiovascular Surgery. 1987;93:173-81.

4. Fuehner T, Kuehn C, Hadem J, Wiesner O, Gottlieb J, Tudorache I, Olsson KM, Greer M, Sommer W, Welte T, Haverich A, Hoeper MM, Warnecke G. Extracorporeal membrane oxygenation in awake patients as bridge to lung transplantation. Am J Respir Crit Care Med. 2012;185:763-8.

5. Maurer JR, Frost AE, Estenne M, Higenbottam T, Glanville AR. INTERNATIONAL GUIDELINES FOR THE SELECTION OF LUNG TRANSPLANT CANDIDATES. Transplantation. 1998;66:951-6.

6. Jurmann MJ, Schaefers HJ, Demertzis S, Haverich A, Wahlers T, Borst HG. Emergency lung transplantation after extracorporeal membrane oxygenation. Asaio j. 1993;39:M448-52.

7. George TJ, Beaty CA, Kilic A, Shah PD, Merlo CA, Shah AS. Outcomes and temporal trends among high-risk patients after lung transplantation in the United States. The Journal of Heart and Lung Transplantation. 2012;31:1182-91.

8. Toyoda Y, Bhama JK, Shigemura N, Zaldonis D, Pilewski J, Crespo M, Bermudez C. Efficacy of extracorporeal membrane oxygenation as a bridge to lung transplantation. Journal of Thoracic and Cardiovascular Surgery. 2013;145:1065-71.

9. Cypel M, Keshavjee S. Extracorporeal life support as a bridge to lung transplantation. Clin Chest Med. 2011;32:245-51.

10. Lafarge M, Mordant P, Thabut G, Brouchet L, Falcoz PE, Haloun A, Le Pimpec-Barthes F, Maury JM, Reynaud-Gaubert M, Saint-Raymond C, Sage E, Stern M, Thomas P, Castier Y, Dorent R, Mal H. Experience of extracorporeal membrane oxygenation as a bridge to lung transplantation in France. $J$ Heart Lung Transplant. 2013;32:905-13.

11. Benazzo A, Schwarz S, Frommlet F, Schweiger T, Jaksch P, Schellongowski P, Staudinger T, Klepetko W, Lang G, Hoetzenecker K. Twenty-year experience with extracorporeal life support as bridge to lung transplantation. J Thorac Cardiovasc Surg. 2019;157:2515-25.e10.

12. Ko Y, Cho YH, Park YH, Lee H, Suh GY, Yang JH, Park CM, Jeon K, Chung CR. Feasibility and Safety of Early Physical Therapy and Active Mobilization for Patients on Extracorporeal Membrane Oxygenation. Asaio j. 2015;61:564-8.

13. Biscotti M, Gannon WD, Agerstrand C, Abrams D, Sonett J, Brodie D, Bacchetta M. Awake Extracorporeal Membrane Oxygenation as Bridge to Lung Transplantation: A 9-Year Experience. Ann 
Thorac Surg. 2017;104:412-9.

14. Langer T, Santini A, Bottino N, Crotti S, Batchinsky Al, Pesenti A, Gattinoni L. "Awake" extracorporeal membrane oxygenation (ECMO): pathophysiology, technical considerations, and clinical pioneering. Crit Care. 2016;20:150.

15. Contentin L, Ehrmann S, Giraudeau B. Heterogeneity in the definition of mechanical ventilation duration and ventilator-free days. Am J Respir Crit Care Med. 2014;189:998-1002.

16. ATS statement: guidelines for the six-minute walk test. Am J Respir Crit Care Med. 2002;166:111-7.

17. Crotti S, Bottino N, Spinelli E. Spontaneous breathing during veno-venous extracorporeal membrane oxygenation. J Thorac Dis. 2018;10:S661-s9.

18. Lehr CJ, Zaas DW, Cheifetz IM, Turner DA. Ambulatory Extracorporeal Membrane Oxygenation as a Bridge to Lung Transplantation. Chest. 2015;147:1213-8.

19. Stahl K, Seeliger B, Hoeper MM, David S. "Better be awake"-a role for awake extracorporeal membrane oxygenation in acute respiratory distress syndrome due to Pneumocystis pneumonia. Crit Care. 2019;23:418.

20. Senger D, Erbguth F. [Critical illness myopathy and polyneuropathy]. Med Klin Intensivmed Notfmed. 2017;112:589-96.

21. Kortebein P, Ferrando A, Lombeida J, Wolfe R, Evans WJ. Effect of 10 days of bed rest on skeletal muscle in healthy older adults. Jama. 2007;297:1772-4.

22. Truong AD, Fan E, Brower RG, Needham DM. Bench-to-bedside review: Mobilizing patients in the intensive care unit - from pathophysiology to clinical trials. Critical Care. 2009;13:216.

23. Andrade FH, Reid MB, Allen DG, Westerblad H. Effect of hydrogen peroxide and dithiothreitol on contractile function of single skeletal muscle fibres from the mouse. J Physiol. 1998;509 ( Pt 2):56575.

24. Pehlivan E, Mutluay F, Balcı A, Kılıç L. The effects of inspiratory muscle training on exercise capacity, dyspnea and respiratory functions in lung transplantation candidates: a randomized controlled trial. Clin Rehabil. 2018;32:1328-39.

25. Rozenberg D, Wickerson L, Singer LG, Mathur S. Sarcopenia in lung transplantation: a systematic review. J Heart Lung Transplant. 2014;33:1203-12.

26. Dierich M, Tecklenburg A, Fuehner T, Tegtbur U, Welte T, Haverich A, Warnecke G, Gottlieb J. The influence of clinical course after lung transplantation on rehabilitation success. Transpl Int. 2013;26:322-30.

27. Yoshida T, Amato MBP, Kavanagh BP, Fujino Y. Impact of spontaneous breathing during mechanical ventilation in acute respiratory distress syndrome. Curr Opin Crit Care. 2019;25:192-8.

28. Goligher EC, Dres M, Fan E, Rubenfeld GD, Scales DC, Herridge MS, Vorona S, Sklar MC, Rittayamai N, Lanys A, Murray A, Brace D, Urrea C, Reid WD, Tomlinson G, Slutsky AS, Kavanagh BP, Brochard LJ, Ferguson ND. Mechanical Ventilation-induced Diaphragm Atrophy Strongly Impacts Clinical Outcomes. Am J Respir Crit Care Med. 2018;197:204-13. 
29. Hakim AH, Ahmad U, McCurry KR, Johnston DR, Pettersson GB, Budev M, Murthy S, Blackstone EH, Tong MZ. Contemporary Outcomes of Extracorporeal Membrane Oxygenation Used as Bridge to Lung Transplantation. Ann Thorac Surg. 2018;106:192-8.

30. Lee JG, Park MS, Jeong SJ, Kim SY, Na S, Kim J, Paik HC. Critical Care before Lung Transplantation. Acute Crit Care. 2018;33:197-205.

31. International Society for $\mathrm{H}$, Lung T, Paik HC. Current perspective of lung transplantation. Journal of the Korean Medical Association. 2016;59:A119-24.

\section{Figures}

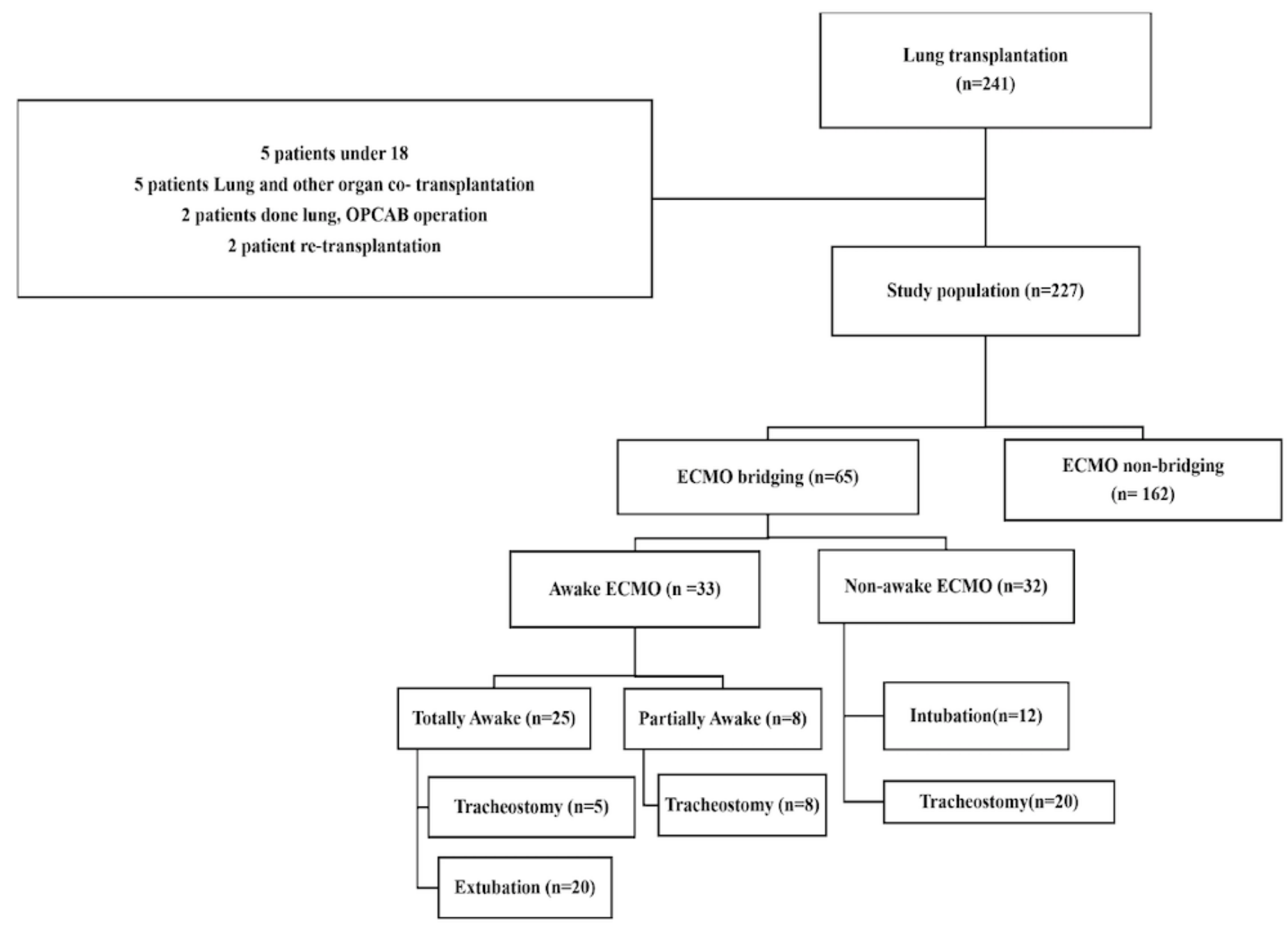

Figure 1 
Study design 227 lung transplant recipients enrolled in this study, among them 65 were bridged with ECMO, 33 were applied awake ECMO strategy.

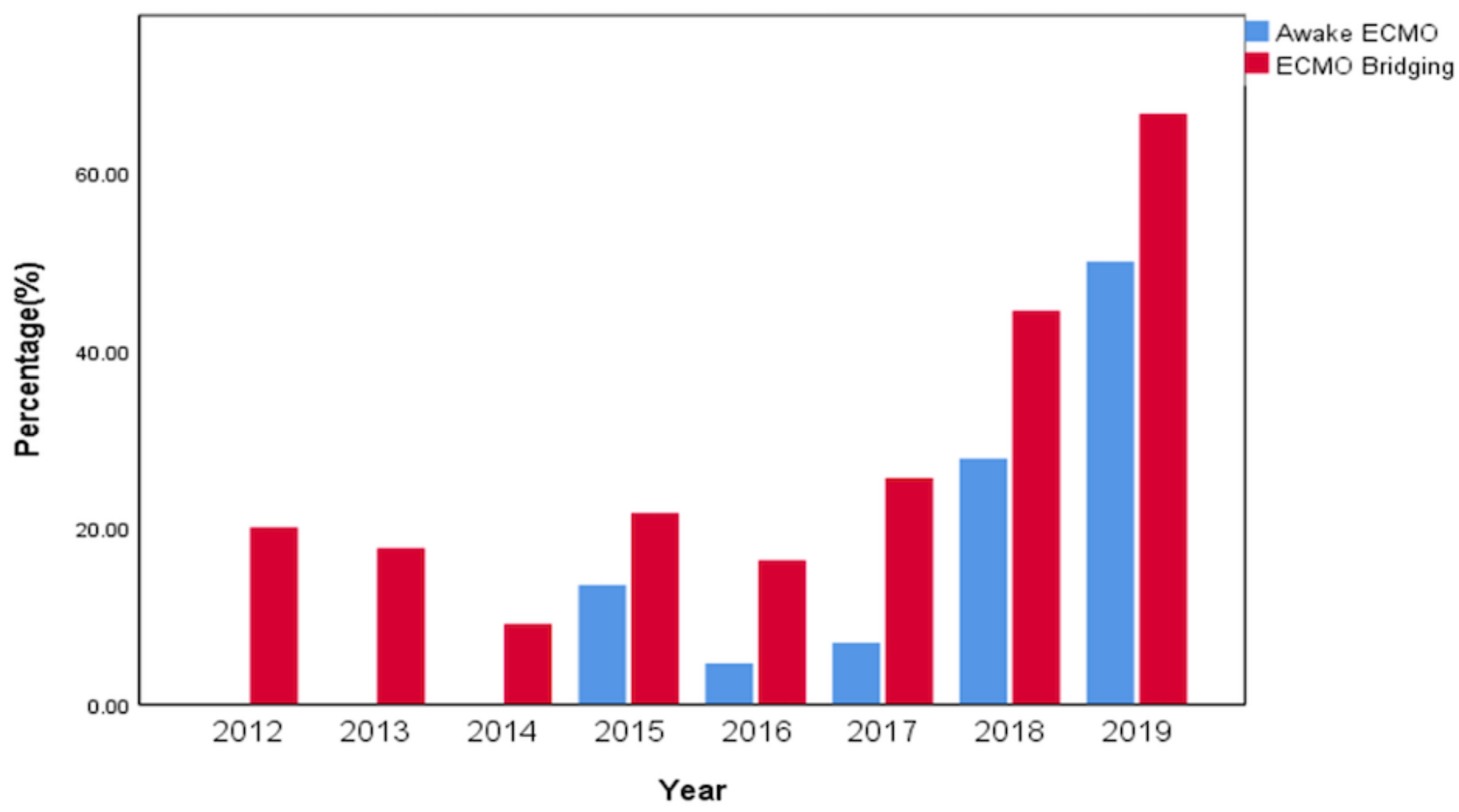

Figure 2

Percentage of Awake ECMO and ECMO bridging in severance hospital Incidence of bridging ECMO is increasing up to $20 \%$ in lung transplant recipients after 2017 , also proportion of awake ECMO is rapidly increasing. 


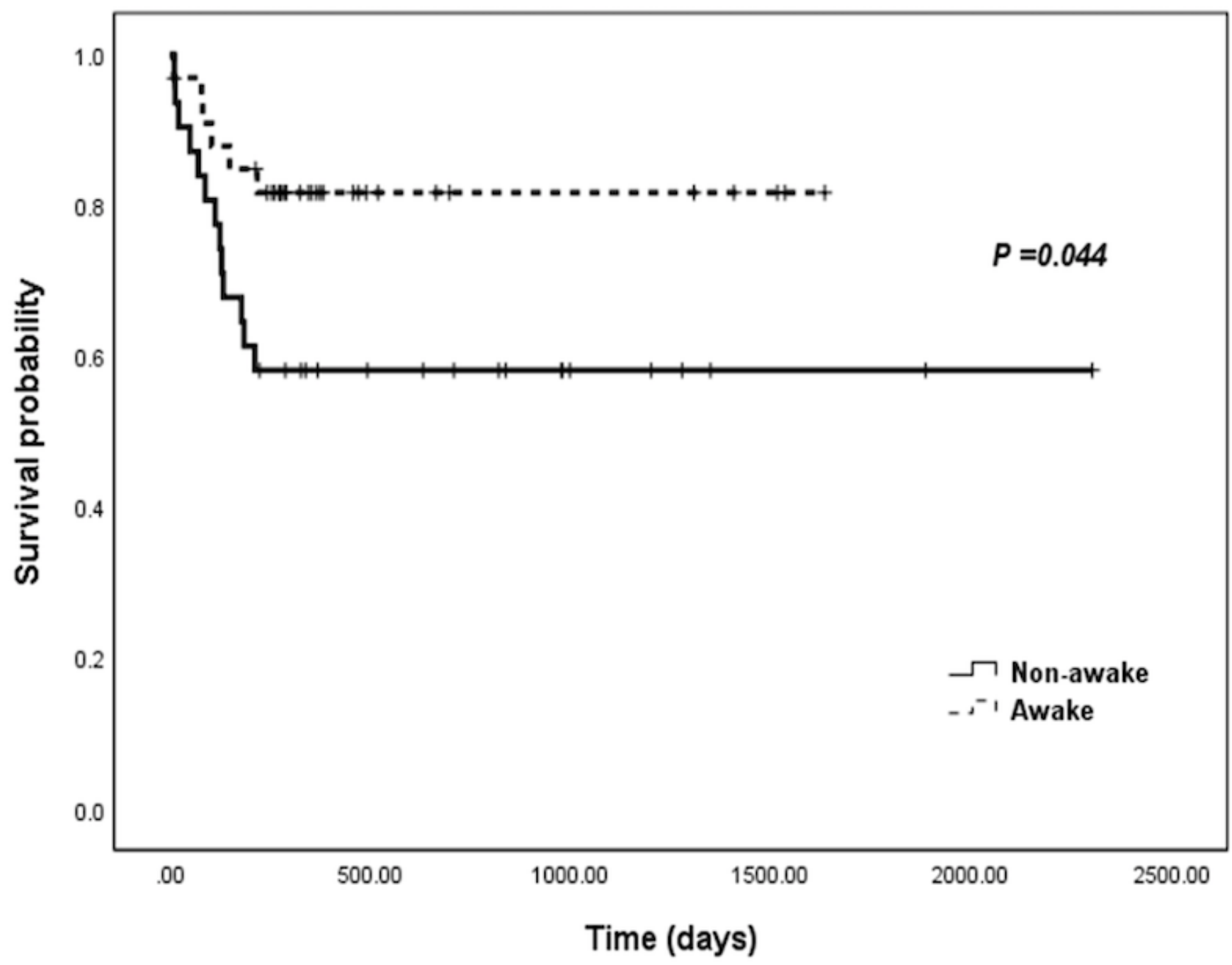

Figure 3

Kaplan-meier survival curves of bridged ECMO patients stratification by Awake strategy This KaplanMeier plot shows statically survival benefit of Awake ECMO compared to non-awake ECMO group. (18.2\% versus $40.6 \%, P=0.04$ )

\section{Supplementary Files}

This is a list of supplementary files associated with this preprint. Click to download.

- SupplementFigure1.tif

- SupplementFigure2.tif 